\title{
HOLOMORPHIC FUNCTIONS WITH POSITIVE REAL PART ON POLYCYLINDERS
}

BY

\author{
A. KORÁNYI(1) AND L. PUKÁNSZKY(1)
}

The purpose of this paper is to generalize the classical Riesz-Herglotz integral representation and Pick-Nevanlinna interpolation theorems to functions of several complex variables. A generalization of these results in a somewhat different direction has already been given in [2]; the functions with positive real part, which will be the objects of our study here, turn out to be closely related to a subclass of the class $H_{m}$ considered in [2].

We note that some of our results (Theorem 1 and the necessity part of Theorem 2) apply to a much larger class of domains than the polycylinders. In fact, the Szegö kernel function, which plays a crucial role in our investigation, exists and has very similar properties in all the bounded homogeneous domains which are starlike, circular, and whose isotropy group is linear and transitive on the BergmanŠilov boundary (cf. [1]; the symmetric domains of E. Cartan are all such). Our proofs apply to this more general situation as well, with the only change that the system (1) has to be replaced by a system of polynomials orthonormal on the Bergman-Šilov boundary and constructed from the irreducible representations of the isotropy group. The holomorphic elements of this system will then span the range of the projection $P$. Our method of proving the second part of Theorem 2 , however, seems not to be applicable here, and so the problem of the sufficiency of our conditions in the general case remains open.

We shall denote by $z=\left(z_{1}, \cdots, z_{m}\right)$ the points of complex Euclidean $m$-space $C^{m}$. $D$ will be the unit polycylinder of $C^{m}$,

$$
D=\left\{z|| z_{k} \mid<1 \quad(k=1, \cdots, m)\right\} .
$$

The Bergman-Šilov boundary of $D$ is the multi-torus

$$
B=\left\{u|| u_{k} \mid=1 \quad(k=1, \cdots, m)\right\} .
$$

Writing $u_{k}=e^{i \phi_{k}}(k=1, \cdots, m), d u=(2 \pi)^{-m} d \phi_{1} \cdots d \phi_{m}$ is the Haar measure of $B$. We shall denote by $L^{2}(B)$ the Hilbert space of square-integrable functions on $B$ with respect to $d u$. The inner product in $L^{2}(B)$ will be denoted by $\langle$,$\rangle . The set of$ functions

$$
\left\{u_{1}^{n_{1}} \cdots u_{m}^{n_{m}} \mid-\infty<n_{1}, \cdots, n_{m}<\infty\right\}
$$

Received by the editors June $8,1962$.

(1) Supported by National Science Foundation Contracts G-19137 and G-19701. 
is a complete orthonormal system in $L^{2}(B)$. A function $f$ on $D \cup B$ will be called a continuous holomorphic function if it is holomorphic on $D$ and continuous on $D \cup B$. The restriction of such a function to $B$ will be denoted by the same letter $f$.

The domain $D$ has a Szegö kernel function $S: D \times D \rightarrow C$ defined by

$$
S(z, w)=\prod_{k=1}^{m} \frac{1}{1-z_{k} \bar{w}_{k}}=\sum_{n_{1}, \ldots, n_{m} \geqq 0} \bar{w}_{1}^{n_{1}} \cdots \bar{w}_{m}^{n_{m}} z_{1}^{n_{1}} \cdots z_{m}^{n_{m}} .
$$

The following properties of $S$ are clear (and well known).

(i) $S(z, w)=S(w, z)$ and $S(z, z)>0$ for all $z, w \in D$.

(ii) The function $S_{w}$ defined by $S_{w}(z)=S(z, w)$ is a continuous holomorphic function on $D \cup B$ for all $w \in D$.

(iii) $f(z)=\left\langle f, S_{z}\right\rangle$ for all continuous holomorphic functions $f$ and all $z \in D$.

(iv) $S(z, 0)=1$ for all $z \in D$.

$P$ will denote the projection in $L^{2}(B)$ onto the subspace spanned by the set $\left\{u_{1}^{n_{1}} \cdots u_{m}^{n_{m}} \mid n_{1}, \cdots, n_{m} \geqq 0\right\}$, and $\bar{P}$ the projection onto the subspace spanned by $\left\{u_{1}{ }^{1} \cdots u_{m}^{n_{m}} \mid n_{1}, \cdots, n_{m} \leqq 0\right\}$. If $f$ is a continuous holomorphic function, then clearly $f \in P L^{2}(B), \bar{f} \in \bar{P} L^{2}(B)$, and $\bar{P} f=f(0)$. We also have $\overline{P f}=\bar{P} \bar{f}$ for all $f \in L^{2}(B)$.

By $Q$ we shall denote the projection onto the subspace spanned by $P L^{2}(B)$ and $\bar{P} L^{2}(B)$. This is the complex subspace spanned by the real parts of continuous holomorphic functions.

Now we prove some further simple properties.

(v) If $f$ is continuous holomorphic, then

for all $z \in D$.

$$
\tilde{f}(0)=\left\langle\bar{f}, S_{z}\right\rangle
$$

In fact, we have

$$
\left\langle\bar{f}, S_{z}\right\rangle=\left\langle\bar{f}, P S_{z}\right\rangle=\left\langle P \bar{f}, S_{z}\right\rangle=\left\langle\bar{f}(0), S_{z}\right\rangle=\bar{f}(0) .
$$

(vi) If $f$ is continuous holomorphic, then

$$
\operatorname{Re} f(0)=\langle\operatorname{Re} f, 1\rangle \text {. }
$$

Proof. By (iii) and (iv), $f(0)=\left\langle f, S_{0}\right\rangle=\langle f, 1\rangle$. By (v), $\bar{f}(0)=\langle\bar{f}, 1\rangle$. Adding these the result follows.

(vii) If $f$ is continuous holomorphic, then

$$
f(z)=i \operatorname{Im} f(0)+\left\langle\operatorname{Re} f, 2 S_{z}-1\right\rangle
$$

for all $z \in D$.

This is clear by adding (iii), (v) and (vi).

(viii) If $f$ is continuous holomorphic, then

$$
S(z, w) f(z)=\left\langle f, S_{z} S_{w}\right\rangle
$$

for all $z, w \in D$. 
Proof. $S_{w} f$ is continuous holomorphic, and using (ii) we have

$$
S(z, w) f(z)=\left(S_{w} f\right)(z)=\left\langle S_{w} f, S_{z}\right\rangle=\left\langle f, S_{w} S_{z}\right\rangle .
$$

(ix) If $f$ is continuous holomorphic, then

$$
\left\langle\operatorname{Re} f, S_{z} S_{w}\right\rangle=S(z, w) \frac{f(z)+\tilde{f}(w)}{2}
$$

for all $z, w \in D$.

Proof. We have

$$
S(z, w) \bar{f}(w)=\bar{S}(w, z) \bar{f}(w)=\overline{\left\langle f, S_{w} S_{z}\right\rangle}=\left\langle\bar{f}, S_{z} S_{w}\right\rangle
$$

(using (viii) in the second equality). Adding this to (viii) the assertion follows.

(x) For all $z, w \in D$,

$$
Q\left(S_{z} S_{w}\right)=S(w, z)\left(S_{z}+S_{w}-1\right) .
$$

Proof. By (vii) and (ix) we have

$$
\begin{aligned}
\left\langle\operatorname{Re} f, S_{z} S_{w}\right\rangle & =\frac{1}{2} S(z, w)\left(\left\langle\operatorname{Re} f, 2 S_{z}-1\right\rangle+\overline{\left\langle\operatorname{Re} f, 2 S_{w}-1\right\rangle}\right) \\
& =S(w, z)\left\langle\operatorname{Re} f, S_{z}+S_{w}-1\right\rangle
\end{aligned}
$$

for all continuous holomorphic $f$. Noting that the $\operatorname{Re} f$ span $Q L^{2}(B)$ and that $S_{z}+S_{w}-1 \in Q L^{2}(B)$ the assertion follows.

We note that by using (viii) we could deduce the sharper results $P\left(S_{z} S_{w}\right)=S(w, z) S_{z}$ and $\bar{P}\left(S_{z} S_{w}\right)=S(w, z) S_{w}$; these, however, will not be used in the sequel.

Our first theorem is a rather straightforward generalization of the RieszHerglotz theorem, we have not, however, been able to find it in the literature. It differs from known integral representations (e.g. L. Bers, Amer. J. Math. 64 (1942), 514-530) by the fact that its kernel is holomorphic, not only biharmonic or harmonic, and that it furnishes a necessary and sufficient condition.

THEOREM 1. The function $f: D \rightarrow C$ is holomorphic and has non-negative real part in $D$ if and only if it admits a representation

$$
f(z)=i \operatorname{Im} f(0)+\int_{B}(2 S(z, u)-1) d \mu(u)
$$

with a positive measure $\mu$ on $B$ such that

$$
\int_{B} u_{1}^{n_{1}} \cdots u_{m}^{n_{m}} d \mu(u)=0
$$

unless $n_{k} \geqq 0$ for all $k=1, \cdots, m$ or $n_{k} \leqq 0$ for all $k=1, \cdots, m$. 
Proof. Let $f$ be holomorphic with non-negative real part in $D$. Then for all $0<r<1$, the function $f_{r}$ defined by $f_{r}(z)=f(r z)$ is continuous holomorphic. By (vii) we have

$$
f_{r}(z)=i \operatorname{Im} f_{r}(0)+\int_{B}(2 S(z, u)-1) d \mu_{r}(u),
$$

where $d \mu_{r}(u)=\operatorname{Re} f_{r}(u) d u$ is a positive measure on $B$. We also have

$$
\int_{B} u_{1}^{n_{1}} \cdots u_{m}^{n_{m}} d \mu_{r}(u)=0
$$

unless $n_{k} \geqq 0$ for all $k$ or $n_{k} \leqq 0$ for all $k$.

For $r \rightarrow 1$ the total variation of $\mu_{r}$ is bounded, and in fact constant, since

$$
\int_{B} d \mu_{r}(u)=\int_{B}(2 S(0, u)-1) \operatorname{Re} f_{r}(u) d u=\operatorname{Re} f_{r}(0)=\operatorname{Re} f(0),
$$

by (iv) and (vii). Hence the well-known theorem of Helly can be applied, and (2) and (3) follow.

Conversely, if $f$ is defined by (1) it is holomorphic in $D$ since the integral converges uniformly and absolutely on compact subsets of $D$. Now note that if $f$ in $L^{2}(B)$ has an absolutely and uniformly convergent expansion in terms of the orthonormal system (1), then by (3) we have

$$
\int_{B} f(u) d \mu(u)=\int_{B}(Q f)(u) d \mu(u)
$$

$S_{z} S_{z}$ is such a function for every fixed $z \in D$. Hence, using (x) we obtain

$$
\begin{aligned}
\operatorname{Re} f(z) & =\int_{B}(S(z, u)+S(z, u)-1) d \mu(u) \\
& =S(z, z)^{-1} \int_{B} Q\left(S_{z} S_{z}\right)(u) d \mu(u) \\
& =S(z, z)^{-1} \int_{B}\left(S_{z} S_{z}\right)(u) d \mu(u) \\
& =S(z, z)^{-1} \int_{B}|S(z, u)|^{2} d \mu(u)>0
\end{aligned}
$$

finishing the proof of the theorem.

COROLlary. The power series

$$
f(z)=\sum_{n_{1}, \ldots, n_{m} \geqq 0} a_{n_{1}, \ldots, n_{m}} z_{1}^{n_{1}} \cdots z_{m}^{n_{m}}
$$

represents a function holomorphic and having non-negative real part in $D$ if 
and only if the function $\phi: J \times \cdots \times J \rightarrow C$ ( $J$ denoting the group of integers) defined by

$$
\phi\left(n_{1}, \cdots, n_{m}\right)= \begin{cases}2 \operatorname{Re} a_{0, \ldots, 0} & \text { if } n_{1}=\cdots=n_{m}=0 \\ a_{n_{1}, \ldots, n_{m}} & \text { if } n_{1}, \cdots, n_{m} \geqq 0, n_{1}+\cdots+n_{m}>0 \\ \bar{a}_{-n_{1}, \ldots,-n_{m}} & \text { if } n_{1}, \cdots, n_{m} \leqq 0, n_{1}+\cdots+n_{m}<0 \\ 0 & \text { otherwise }\end{cases}
$$

is positive definite.

Proof. If $f$ is holomorphic and has non-negative real part, then

$$
f(z)=i \operatorname{Im} f(0)+2 \sum_{n_{1}, \ldots, n_{m} \geqq 0} z_{1}^{n_{1}} \cdots z_{m}^{n_{m}} \int_{B} \bar{u}_{1}^{n_{1}} \cdots \bar{u}_{m}^{n_{m}} d \mu(u)-\int_{B} d \mu(u)
$$

for all $z \in D$, by (2). Hence, using (3) we have

$$
\phi\left(n_{1}, \cdots, n_{m}\right)=2 \int_{B} u_{1}^{n_{1}} \cdots u_{m}^{n_{m}} d \mu(u)
$$

for all $n_{1}, \cdots, n_{m}$, and thus $\phi$ is positive-definite.

Conversely, if $\phi$ is positive-definite then by the Herglotz-Bochner-Weil theorem there exists a positive measure $\mu$ such that (4) holds. This in turn implies (2) and (3).

THEOREM 2. Let $E \subset D$ be a set such that: $(\alpha)$ there exist numbers $z_{1}^{(0)}, \cdots, z_{m}^{(0)}$ such that $\left(z_{1}, \cdots, z_{m}\right) \in E$ implies $\left(z_{1}, \cdots, z_{k-1}, z_{k}^{(0)}, z_{k+1}, \cdots, z_{m}\right) \in E$ for all $k=1, \cdots, m$, and $(\beta) f$ continuous holomorphic on $D$ and $f(z)=0$ for all $z \in E$ implies $f(z)=0$ for all $x \in D$. Then a function $f: E \rightarrow C$ can be extended to a function $f: D \rightarrow C$ holomorphic and having non-negative real part in $D$ if and only if the function $K: E \times E \rightarrow C$ defined by

$$
K(z, w)=S(z, w) \frac{f(z)+\bar{f}(w)}{2}
$$

is positive-definite.

Proof. Let $f$ be holomorphic on $D$ and such that $\operatorname{Re} f \geqq 0$. For $0<r<1$ consider the function $K_{r}: E \times E \rightarrow C$ defined by

$$
K_{r}(z, w)=S(z, w) \frac{f_{r}(z)+\tilde{f}_{r}(w)}{2} .
$$

Let $z^{(1)}, \cdots, z^{(n)} \in D$ and $a_{1}, \cdots, a_{n} \in C$. Using (ix) we have

$$
\begin{aligned}
\sum_{j, k} K_{r}\left(z^{(j)}, z^{(k)}\right) a_{j} \bar{a}_{k} & =\sum_{j, k}\left\langle\operatorname{Re} f_{r}, S_{z}(j) \bar{S}_{z}(k)\right\rangle a_{j} \bar{a}_{k} \\
& =\int_{B}\left|\sum_{j} a_{j} S\left(z^{(j)}, u\right)\right|^{2} \operatorname{Re} f_{r}(u) d u \geqq 0 .
\end{aligned}
$$


Since this holds for all $0<r<1$ and since $K_{r}(z, w)$ is a continuous function of $r$ for all fixed $z, w \in D$, it follows that

$$
\sum_{j, k} K\left(z^{(j)}, z^{(k)}\right) a_{j} \bar{a}_{k} \geqq 0,
$$

i.e., $K$ is positive-definite.

In proving the converse we first assume that $z_{1}^{(0)}=\cdots=z_{m}^{(0)}=0$. Since $K$ is positive-definite, by a well-known construction there exists a Hilbert space $\mathfrak{S}$ and a set of elements $\left\{e_{z} \mid z \in E\right\}$ in $\mathfrak{H}$ such that

$$
\left\langle e_{z}, e_{w}\right\rangle=K(z, w)
$$

for all $z, w \in E$.

For $1 \leqq k \leqq m$ and $z=\left(z_{1}, \ldots, z_{m}\right)$ we define $k z=\left(z_{1}, \cdots, z_{k-1}, 0, z_{k+1}, \cdots z_{m}\right)$. Now for $e_{z}$ such that $z_{k} \neq 0$ we define

$$
e_{z}(k)=\frac{1}{z_{k}}\left(e_{z}-e_{k z}\right)
$$

If $z_{k}, w_{k} \neq 0$, we have

$$
\begin{aligned}
\left\langle e_{z}(k), e_{w}(k)\right\rangle= & \frac{1}{z_{k} \bar{w}_{k}}\left\langle e_{z}-e_{k z}, e_{w}-e_{k w}\right\rangle \\
= & \frac{1}{z_{k} \bar{w}_{k}}\left(\frac{f(z)+\bar{f}(w)}{2 \prod_{l}\left(1-z_{l} \bar{w}_{l}\right)}-\frac{f(k z)+\bar{f}(w)}{2 \prod_{l \neq k}\left(1-z_{l} \bar{w}_{l}\right)}\right. \\
& \left.-\frac{f(z)+\bar{f}(k w)}{2 \prod_{l \neq k}\left(1-z_{l} \bar{w}_{l}\right)}+\frac{f(k z)+\tilde{f}(k w)}{2 \prod_{l \neq k}\left(1-z_{l} \bar{w}_{l}\right)}\right) \\
= & \frac{f(z)+\bar{f}(w)}{2 \prod_{l}\left(1-z_{l} \bar{w}_{l}\right)}=K(z, w)=\left\langle e_{z}, e_{w}\right\rangle .
\end{aligned}
$$

It follows that the operator $V_{k}$ defined on the set $\mathfrak{M}_{k}$ of finite linear combinations of the $e_{z}\left(z_{k}=0\right)$ by

$$
V_{k} \sum_{z} a_{z} e_{z}=\sum_{z} a_{z} e_{z}(k)
$$

is well defined and isometric. By closure and by setting it equal to 0 on the orthogonal complement of $\mathfrak{M}_{k}, V_{k}$ can be extended to an operator $\tilde{V}_{k}$ defined on $\mathfrak{S}$ and such that $\left\|\tilde{V}_{k}\right\| \leqq 1$. Then the operator $\left(I-z_{k} \tilde{V}_{k}\right)^{-1}$ exists and represents a holomorphic operator-valued function for $\left|z_{k}\right|<1$. By (7) we have

$$
e_{z}=\left(I-z_{k} \tilde{V_{k}}\right)^{-1} e_{k z}
$$

whenever $z_{k} \neq 0$; this relation, however, is trivially true if $z_{k}=0$, hence it holds for all $z \in E$. 
It follows that (denoting $0=(0, \cdots, 0))$

$$
e_{z}=\prod_{k=1}^{m}\left(I-z_{k} \tilde{V}_{k}\right)^{-1} e_{0}
$$

Now by (iv) we have

and

$$
\begin{aligned}
\frac{f(z)+\tilde{f}(0)}{2} & =S(z, 0) \frac{f(z)+\tilde{f}(0)}{2}=\left\langle e_{z}, e_{0}\right\rangle \\
& =\left\langle\prod_{k=1}^{m}\left(I-z_{k} \tilde{V}_{k}\right)^{-1} e_{0}, e_{0}\right\rangle,
\end{aligned}
$$

$$
f(z)=-\tilde{f}(0)+2\left\langle\prod_{k=1}^{m}\left(I-z_{k} \widetilde{V}_{k}\right)^{-1} e_{0}, e_{0}\right\rangle
$$

for all $z \in E$. Now the function $\tilde{f}$ defined for all $z \in D$ by the same formula (9) is clearly holomorphic in $D$.

It remains to show that $\operatorname{Re} \tilde{f} \geqq 0$ in $D$. This can be be done as follows. We define the functions $\widetilde{K}$ and $R$ on $D \times D$ by

$$
\widetilde{K}(z, w)=S(z, w) \frac{\tilde{f}(z)+\text { conj. } \tilde{f}(w)}{2}
$$

and

$$
\hat{K}(z, w)=\left\langle\prod_{k=1}^{m}\left(I-z_{k} \tilde{V}_{k}\right)^{-1} e_{0}, \prod_{k=1}^{m}\left(I-w_{k} V_{k}\right)^{-1} e_{0}\right\rangle .
$$

By (5), (6) and (8), $\widetilde{K}$ and $\tilde{K}$ coincide on $E \times E$. Since both are holomorphic in $z$ and antiholomorphic in $w$, an application of the condition $(\beta)$ gives that they are identical also on $D \times D$. Thus, for all $z \in D$,

$$
\begin{aligned}
\operatorname{Re} \tilde{f}(z) & =S(z, z)^{-1} \tilde{K}(z, z)=S(z, z)^{-1} \hat{K}(z, z) \\
& =S(z, z)^{-1}\left\|\prod_{k=1}^{m}\left(I-z_{k} V_{k}\right)^{-1} e_{0}\right\|^{2} \geqq 0,
\end{aligned}
$$

which was to be shown.

Finally we show that the general case can be reduced to the case where $z_{1}^{(0)}=\ldots=z_{m}^{(0)}=0$. We can always find a holomorphic one-to-one mapping $\phi$ of $D$ onto itself such that $\phi:\left(z_{1}^{(0)}, \cdots, z_{m}^{(0)}\right) \rightarrow(0, \cdots, 0)$ and $\phi$ is a fractional linear transformation in each coordinate. Then a simple explicit computation shows that the function $g=f \circ \phi^{-1}$ defined on $\phi(E)$ satisfies the conditions of Theorem 2, and hence by the first part of our proof can be extended to a function $\tilde{g}$ holomorphic on $D$ and such that $\operatorname{Re} \tilde{g} \geqq 0 . \tilde{f}=\tilde{g} \circ \phi$ will then be the desired extension of $f$, and the proof of the theorem is complete.

In conclusion we add some remarks.

1. With the aid of Lemma 6 in [2], Theorem 2 can easily be generalized to the 
case of functions $f$ whose values are operators on a Hilbert space $\mathfrak{H}$. The proof makes use of our Theorem 1 and follows the argument of Theorem 5 in [2].

2. Let $H$ denote the half-plane $\operatorname{Re} z>0$. The Szegö kernel function $S_{H}$ of $H$ is given by $S_{H}(z, w)=(2 \pi)^{-1}(z+\bar{w})^{-1}$. Thus condition (5) can be restated as the positive definiteness of the function

$$
\frac{S(z, w)}{S_{H}(f(z), f(w))} \text {. }
$$

By a computation using fractional linear transformations one can prove that if $H$ is any circular domain in the complex plane and $S_{H}$ is its Szegö kernel, then under the conditions of Theorem 2 the positive definiteness of (10) is necessary and sufficient for the existence of a holomorphic extension $\tilde{f}: D \rightarrow H$ of $f$.

3. It is clear from the proof of Theorem 2 that a holomorphic extension of $f$ exists even if hypothesis $(\beta)$ is not assumed, only it will not necessarily have nonnegative real part. For this latter to be true $(\beta)$ is essential. We give here an example of a set $E$ and a function $f: E \rightarrow C$ such that (5) is positive-definite, but $f$ does not have an extension of the desired kind.

Choose the numbers $r, s$ in such a way that

$$
\begin{gathered}
0<r<s<1, \\
\left(1-r^{2}\right)^{2}<1-s^{2} .
\end{gathered}
$$

Let $D$ be the bicylinder, let $E=\{(0,0),(r, r)\}$, and let $f$ be defined by

$$
\begin{aligned}
& f(0,0)=1 \\
& f(r, r)=\frac{1+s}{1-s} .
\end{aligned}
$$

It is easily seen that (5) is positive-definite. However, if there exists a holomorphic extension $\tilde{f}$ of $f$ with $\operatorname{Re} \tilde{f} \geqq 0$, then the function $g$ defined by

$$
g(\zeta)=\frac{\tilde{f}(\zeta, \zeta)-1}{\tilde{f}(\zeta, \zeta)+1}
$$

is holomorphic such that $|g(\zeta)| \leqq 1$ for all $|\zeta|<1$, while $g(0)=0$ and $g(r)=s$ in contradiction to the Schwarz lemma.

\section{REFERENCES}

1. L.-K. Hua, Harmonic analysis of functions of several variables in the classical domains, Moscow, 1959. (Russian)

2. A. Korányi, On some classes of analytic functions of several variables, Trans. Amer. Math. Soc. 101 (1961), 520-554.

UNIVERSITY OF CALIFORNIA, Berkeley, CALIForNia

STANFORD UNIVERSITY, Stanford, CALIFORNIA 